\begin{tabular}{llll} 
Abstract G414(P) Table 1 & \multicolumn{3}{c}{ Results of data collection } \\
\hline & Patient Numbers & Mean HR & Confidence \\
Treatment group & $(\mathbf{n}=\mathbf{4 7})$ & Reduction & Interval \\
High dose dexamethasone & 14 & $45.1 \%$ & $39.1 \%-51.0 \%$ \\
Standard dose dexamethasone & 23 & $42.9 \%$ & $38.5 \%-47.4 \%$ \\
Prednisolone & 4 & $48.0 \%$ & $33.9 \%-62.1 \%$ \\
Other & 6 & $36.3 \%$ & $29.9 \%-42.6 \%$ \\
\hline
\end{tabular}

Results Total of 136 patients were diagnosed, 6 notes were not located. 60/130 had steroid treatment. 47/60 (78.3\%) experienced significant bradycardia (Table 1).

There was no statistically significant difference in the degree of bradycardia between the different dosages and types of steroids used.

Infants were not at a significantly higher risk for bradycardia $(n=3)$.

ECG on $11 / 47$ patients showed either no change or sinus bradycardia. IOP was raised in $2 / 3$ measured patients.

Conclusions This is the first clinical study reviewing the clinical relevance of steroid induced bradycardia in paediatric cancer.

Majority of patients on steroid treatment for cancer showed significant bradycardia with no clinical decompensation. No risk factors were identified with respect to dose, type of steroid or age group.

We recommend correlation with BP, ECG, probably IOP measurement in the monitoring of patients on steroid treatment for cancer chemotherapy. Appropriate PEWS charting and escalation of management should still be followed.

This is also the first study to document clinically relevant steroid induced glaucoma in paediatric malignancy treatment. This has led to a further collaborative study to investigate the interaction/mechanism of steroid induced glaucoma and bradycardia in paediatric malignancy.

\section{G415(P) CYTOMEGALOVIRUS INFECTION PRESENTING AS RECURRENT FEVER IN A CHILD RECEIVING STANDARD CHEMOTHERAPY FOR ACUTE LYMPHOBLASTIC LEUKAEMIA}

${ }^{1} \mathrm{~K}$ Macdonald, ${ }^{2} \mathrm{M}$ Cummins, ${ }^{1} \mathrm{~S}$ Goyal. ${ }^{1}$ CLIC Unit, Royal Cornwall Hospitals NHS Trust, Truro, UK; ${ }^{2}$ Department of Paediatric Haematology, Bristol Royal Hospital for Children, Bristol, UK

\subsection{6/archdischild-2015-308599.369}

Introduction Infection presenting with fever is a common cause for hospital admission of immunocompromised children. Initial management is aimed at the treatment and identification of bacterial and fungal infection. Cytomegalovirus (CMV) is well recognised as causing increased morbidity and mortality in immunocompromised children who have undergone stem cell transplants but less so in children receiving standard chemotherapy in acute lymphoblastic leukaemia.

Case description Our patient is a 7 year old girl receiving standard chemotherapy for acute lymphoblastic leukaemia. Post induction she was admitted with significant fever but no other clinical signs or symptoms. She was not neutropenic, CRP was unremarkable and cultures negative. A chest X-ray showed right middle lobe changes and CT chest showed consolidation and atelectasis but no ground glass appearance or nodules. Candida PCR and beta-D-glucan test were negative. She received a course of empiric antibiotics, including macrolides, and antifungal treatment. Her fever settled. Following cessation of treatment she was readmitted with fever and a dry cough and intravenous antibiotic and antifungal therapy was recommenced. Repeat investigative workup was unremarkable. She settled. A further brief admission with fever settled without intervention following which she was readmitted for the fourth time with fever and cough. She became unwell, developing an oxygen requirement with respiratory signs on chest auscultation. Blood viral PCRs were sent. CMV PCR was significantly elevated at 142,000 copies/ml. BAL fluid was CMV positive and negative for fungi. Intravenous foscarnet was added to the treatment regime. She improved with this and empirical antifungal treatment.

Results and conclusion This patient presented with recurrent fevers resulting in prolonged admissions impacting on quality of life and interruption of treatment. No other positive pathogens for the fever were identified apart from CMV. A literature review confirms that $\mathrm{CMV}$ is an opportunistic infection that can cause serious, sometimes life-threatening, illness even during conventional anticancer infection due to profound immunosuppression. This is less recognised in clinical practice. We would highlight the importance of considering CMV infection in cases of recurrent and prolonged fever in these children.

\section{G416(P) HYPOGLYCAEMIA SECONDARY TO 6-MERCAPTOPURINE IN LEUKAEMIA TREATMENT}

${ }^{1} \mathrm{~L}$ Roe, ${ }^{1} \mathrm{~K}$ Neuling, ${ }^{2} \mathrm{~A}$ Dunlop. ${ }^{1}$ Paediatrics, University Hospital Coventry and Warwickshire, Coventry, UK; ${ }^{2}$ Library Information, University Hospital Coventry and Warwickshire, Coventry, UK

\subsection{6/archdischild-2015-308599.370}

Aims To highlight a significant side effect of a chemotherapy drug that is currently poorly recognised in the UK. Hypoglycaemia is a rare but potentially serious side effect of 6-mercaptopurine that forms an integral part of current maintenance chemotherapy for children with acute lymphoblastic leukaemia (ALL). There are now multiple well documented cases in the literature from the USA, Italy and Finland. However in the UK it does not feature in the drug information. This means it is not being discussed with families as a potential side effect to look out for in their children.

Methods We reviewed the case notes and results of 3 children who over the past 5 years were receiving maintenance therapy for ALL and developed symptomatic hypoglycaemia. A literature review was performed looking at hypoglycaemia in children on chemotherapy for ALL and possible risk factors and management strategies that have been identified.

Results We describe 3 cases of symptomatic hypoglycaemia occurring within aUKoncology shared care department after varying periods of fasting. The children were aged 4 years 8 months, 5 years 2 months and 8 years 4 months. Other causes of hypoglycaemia were excluded. On further investigation other unprovoked episodes were identified in these children and strategies put in place to try and prevent and manage them. The children all required treatment in the form of oral or IV glucose at their initial presentation. The hypoglycaemic episodes ceased once the chemotherapy was completed.

Conclusion Symptomatic hypoglycaemia remains a rare but real risk in children receiving maintenance chemotherapy for ALL. Relatively short periods of starvation have been known to cause hypoglycaemia. These children are regularly fasted for procedures such as lumbar punctures and bone marrow aspirates it is essential that the medical and nursing teams are aware of the 